\title{
Location-Aware Topology Matching in P2P Systems
}

\author{
Yunhao Liu, Xiaomei Liu, Li Xiao \\ Dept. of Computer Science and Engineering \\ Michigan State University \\ East Lansing, MI 48824 \\ \{liuyunha, liuxiaom, lxiao\}@cse.msu.edu
}

\author{
Lionel M. Ni \\ Dept. of Computer Science \\ Hong Kong Univ. of Science and Technology \\ Kowloon, Hong Kong \\ ni@cs.ust.hk
}

\author{
Xiaodong Zhang \\ Dept. of Computer Science \\ College of William and Mary \\ Williamsburg, VA 23187 \\ zhang@cs.wm.edu
}

\begin{abstract}
Peer-to-Peer (P2P) computing has emerged as a popular model aiming at further utilizing Internet information and resources, complementing the available client-server services. However, the mechanism of peers randomly choosing logical neighbors without any knowledge about underlying physical topology can cause a serious topology mismatching between the P2P overlay network and the physical underlying network. The topology mismatching problem brings a great stress in the Internet infrastructure and greatly limits the performance gain from various search or routing techniques. Meanwhile, due to the inefficient overlay topology, the flooding-based search mechanisms cause a large volume of unnecessary traffic. Aiming at alleviating the mismatching problem and reducing the unnecessary traffic, we propose a locationaware topology matching (LTM) technique, an algorithm of building an efficient overlay by disconnecting low productive connections and choosing physically closer nodes as logical neighbors while still retaining the search scope and reducing response time for queries. LTM is scalable and completely distributed in the sense that it does not require any global knowledge of the whole overlay network when each node is optimizing the organization of its logical neighbors. The effectiveness of LTM is demonstrated through simulation studies.
\end{abstract}

Keywords-peer-to-peer; topology mismatching; blind flooding; location-aware topology matching; search efficiency

\section{INTRODUCTION}

There are mainly three different architectures for P2P systems: centralized, decentralized structured, and decentralized unstructured [18]. In centralized model, such as Napster [6], central index servers are used to maintain a directory of shared files stored on peers so that a peer can search for the whereabouts of a desired content from an index server. However, this architecture creates a single point of failure, and its centralized nature of the service also makes systems vulnerable to denial of service attacks [15]. Decentralized P2P systems have the advantages of eliminating reliance on central servers and providing greater freedom for participating users to exchange information and services directly between each other. In decentralized structured models, such as Chord [33], Pastry [27], Tapestry [38], and CAN [24], the shared data placement and topology characteristics of the network are tightly controlled based on distributed hash functions.

This paper focuses on decentralized unstructured P2P systems, such as Gnutella [3] and KaZaA [5]. File placement is random in these systems, which has no correlation with the network topology [37]. Unstructured P2P systems are most commonly used in today's Internet. The most popular search mechanism in use is to blindly "flood" a query to the network among peers (such as in Gnutella) or among supernodes (such as in $\mathrm{KaZaA}$ ). A query is broadcast and rebroadcast until a certain criterion is satisfied. If a peer receiving the query can provide the requested object, a response message will be sent back to the source peer along the inverse of the query path. This mechanism ensures that the query will be "flooded" to as many peers as possible within a short period of time in a P2P overlay network. A query message will also be dropped if the query message has visited the peer before.

Studies in [31] and [29] have shown that P2P traffic contributes the largest portion of the Internet traffic based on their measurements on some popular P2P systems, such as FastTrack (including KaZaA and Grokster) [2], Gnutella, and DirectConnect. Measurements in [25] have shown that even $95 \%$ of any two nodes are less than 7 hops away, the flooding-based routing algorithm generates $330 \mathrm{~TB} /$ month in a Gnutella network with only 50,000 nodes. A large portion of the heavy P2P traffic caused by inefficient overlay topology and the blind flooding is unnecessary, which makes the unstructured P2P systems being far from scalable [26]. There are three reasons for this problem. First, the mechanism of a peer randomly choosing logical neighbors without any knowledge about the underlying physical topology causes topology mismatching between the P2P logical overlay network and physical underlying network. Because of the mismatch problem, the same message may traverse the same physical link multiple times, causing a large amount of unnecessary traffic. Second, a query may be flooded to multiple paths that are merged to the same peer. In this case, only the traffic along one of the paths is necessary. Finally, two neighboring peers may forward the same query message to each other before they receive the query message from each other. Thus, the same query message may traverse the same logical link twice.

Aiming at alleviating the mismatch problem, reducing the unnecessary traffic, and addressing the limits of existing solutions, we propose a location-aware topology matching (LTM) scheme, in which each peer issues a detector in a small region so that the peers receiving the detector can record relative delay information. Based on the delay information, a receiver can detect and cut most of the inefficient and redundant logical links, and add closer nodes as its direct neighbors. Our simulation studies show that the total traffic

This work was partially supported by the US National Science Foundation (NSF) under grant CCR-0098055, ACI-0129883, and ACI-0325760, by Michigan State University IRGP Grant 41114, and by Hong Kong RGC Grant HKUST6161/03E. 
and response time of the queries can be significantly reduced by LTM without shrinking the search scope. We also show that the overhead of issuing detectors is trivial compared with the query cost savings.

Our proposed LTM can be used to complement other search techniques, such as forwarding-based search mechanisms [37] or cache-based schemes [14, 19, 20] that will be discussed in Section II. In this paper, we will show this effectiveness by a case study of combining LTM and response index caching scheme, in which query responses are cached in passing peers along the returning path. Our study shows that only one tenth of original traffic cost is necessary to cover the same number of peers, and the average response time is reduced by around $80 \%$.

The rest of the paper is organized as follows. Section II discusses related work. Section III presents the locationaware topology matching (LTM) scheme. Section IV describes our simulation methodology. Performance evaluation of the LTM is presented in Section V, and we conclude the work in Section VI.

\section{RELATED WORK}

Many efforts have been made to avoid the large volume of unnecessary traffic incurred by the flooding-based search in decentralized unstructured P2P systems. In general, three types of approaches have been proposed to improve search efficiency in unstructured P2P systems: forwarding-based, cache-based, and overlay optimization. The three different kinds of approaches can be used together to complement each other.

In forwarding-based approaches, instead of relaying the query messages to all its logical neighbors except the incoming peer, a peer selects a subset of its neighbors to relay the query. In Directed BFS proposed in [37], each peer maintains statistic information based on some metrics, such as the number of results received from neighbors from previous queries or the latency of the connection with that neighbor. A peer selects a subset of the neighbors, such as the neighbors that have returned the largest number of results from previous queries, or the neighbors that have low latency, to send its query. A k-walker query algorithm is proposed in [18], in which a query is sent to $k$ different walkers (relay neighbors) from the source peer. For a peer in each walker, it just randomly selects one neighbor to relay the query. For each walker, the query processing is done sequentially. A hybrid periodical flooding (HPF) approach proposed in [39] improves the search efficiency by selecting forwarding neighbors based on multiple metrics and addressing the partial coverage problem to balance the search cost and response time.

The second approach is cache-based including data index caching and content caching. Centralized P2P systems provide centralized index servers to keep indices of shared files of all peers. KaZaA utilizes cooperative superpeers, each of which is an index server of a subset of peers. Some systems distribute the function of keeping indices to all peers [20]. In Local Indices policy [37], each peer maintains an index of files available in the nodes within given hops of itself. When a peer receives a query, it can process the query on behalf of all nodes within the given hops of itself. Having observed the locality of queries, the authors in $[19,32]$ further propose that each peer cache query strings and results that flow through it. Three different strategies to replicate data (file content or query responses) on multiple peers have been evaluated in [14]. The three strategies are different on the ratio of allocations according to the ratio of query rates. Transparent query caching [22] is proposed to cache query hits at a gateway of an organization based on an observation of query locality in peers within the gateway. Caching file contents has also been studied. For example, an ideal cache (infinite capacity and no expiration) simulator [29] is built for KaZaA P2P traffic to cache file contents, which has shown that caching would have a large effect on a wide-scale P2P system on reducing traffic volume and bandwidth demands.

The third approach is based on overlay topology optimization that is closely related to what we are presenting in this paper. Here we briefly introduce three types of solutions and their comparisons with our approach. End system multicast, Narada, is proposed in [13], which first constructs a rich connected graph on which to further construct shortest path spanning trees. Each tree rooted at the corresponding source using well-known routing algorithms. This approach introduces large overhead of forming the graph and trees in a large scope, and does not consider the dynamic joining and leaving characteristics of peers. The overhead of Narada is proportional to the multicast group size. Our proposed LTM is easy to implement and adaptive to the dynamic nature of $\mathrm{P} 2 \mathrm{P}$ systems with the overhead that is only proportional to the square of the average number of neighbors. Researchers have also considered to cluster close peers based on their IP addresses (e.g., [17, 21]). We believe there are two limitations for this approach. First, the mapping accuracy is not guaranteed by this approach. Second, this approach may affect the searching scope in P2P networks. In contrast, our technique is measurement based and can accurately and dynamically connect the physically closer peers, and disconnect physically distant peers. Furthermore, our scheme does not shrink the search scope. Recently, researchers in [36] have proposed to measure the latency between each peer to multiple stable Internet servers called "landmarks". The measured latency is used to determine the distance between peers. This measurement is conducted in a global P2P domain. In contrast, our measurement is conducted in many small regions, significantly reducing the network traffic with high accuracy. Gia [12] introduced a topology adaptation algorithm to ensure that high capacity nodes are indeed the ones with high degree and low capacity nodes are within short reach of high capacity nodes. It addresses a different matching problem in overlay networks, but does not address the topology mismatching problem between the overlay and physical networks. 


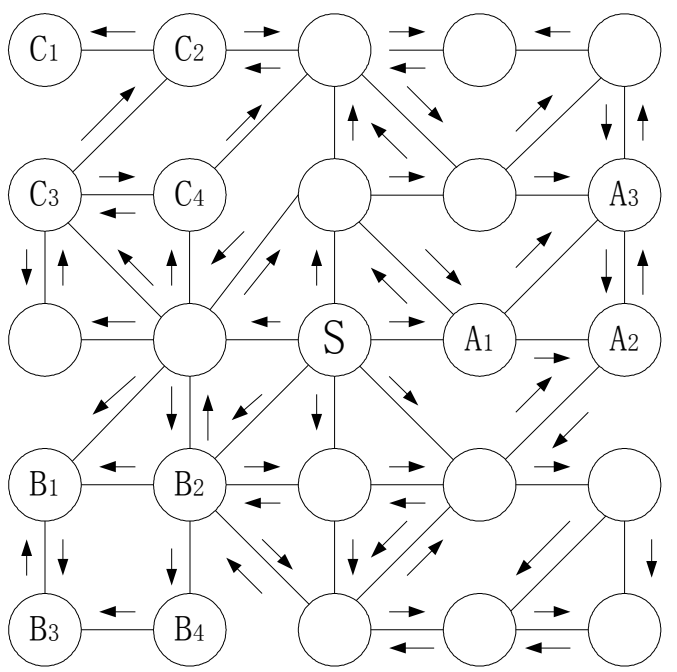

Figure 1. An example of P2P overlay
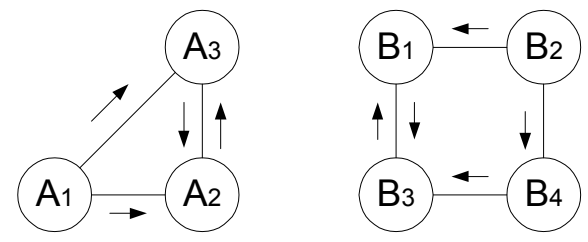

Figure 2. Unnecessary traffic on logical link A2A3 and B1B3

\section{LOCATION-AWARE TOPOLOGY MATCHING}

In a $\mathrm{P} 2 \mathrm{P}$ system, all participating peers form a $\mathrm{P} 2 \mathrm{P}$ network over a physical network. A P2P network is an abstract, logical network called an overlay network. Maintaining and searching operations of a Gnutella peer are specifically described in [4]. When a new peer wants to join a P2P network, a bootstrapping node provides the IP addresses of a list of existing peers in the P2P network. The new peer then tries to connect with these peers. If some attempts succeed, the connected peers will be the new peer's neighbors. Once this peer connects into a P2P network, the new peer will periodically ping the network connections and obtain the IP addresses of some other peers in the network. These IP addresses are cached by this new peer. When a peer leaves the P2P network and then wants to join the P2P network again (no longer the first time), the peer will try to connect to the peers whose IP addresses have already been cached. The mechanism that a peer joins a P2P network, the fact of a peer randomly joining and leaving, and the nature of flooding search make an inefficient mismatched overlay network and cause large amount of unnecessary traffic. In this section, we first use examples to explain the unnecessary traffic and the mismatching problem. We then describe our proposed LTM technique in detail.
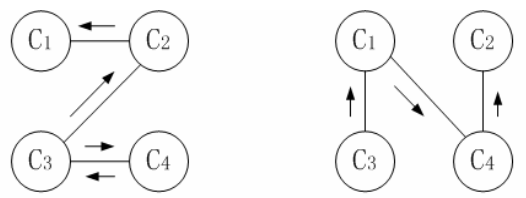

(a) Inefficent Overlay (b) Efficient Overlay

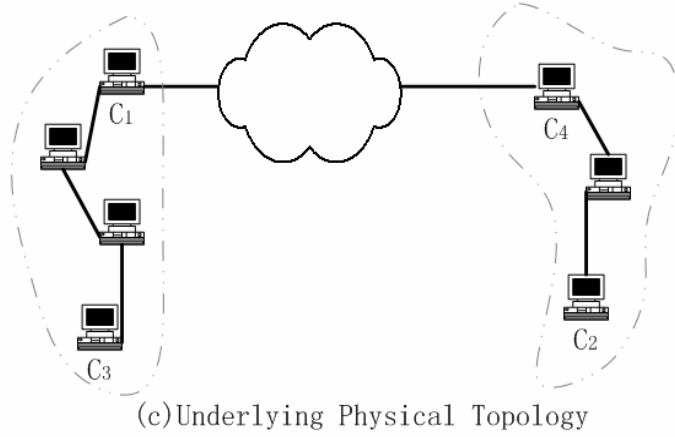

Figure 3. Topology mismatching problem

\section{A. Unnecessary Traffic}

Figure 1 shows an example of a P2P overlay topology where solid lines denote overlay connections among logical P2P neighbors. Consider the case when node S sends a query. A solid arrow represents a delivery of the query message along one logical connection. The query is relayed by many peers, which incurs a lot of unnecessary traffic. Figure 2 shows two subsets of the P2P overlay in Figure 1. In the left of Figure 2, after $A_{1}$ forwards the query to $A_{2}$ and $A_{3}$, if none of $A_{2}$ or $A_{3}$ knows the other one will receive the same query from A1, they will forward the query to each other. The pair of transmission between $\mathrm{A}_{2}$ and $\mathrm{A}_{3}$ is unnecessary. Similarly, in the right of Figure 2, $\mathrm{B}_{3}$ may receive the same query message twice, and depending on the delay of the connections, $\mathrm{B}_{3}$ will forward the message to $B_{1}$ if it receives $B_{4}$ 's message earlier than the same message from $B_{1}$. In these cases, it is clear that the search scope of the query from node $S$ will not shrink without logical connections of $\mathrm{A}_{2} \mathrm{~A}_{3}$ and $\mathrm{B}_{1} \mathrm{~B}_{3}$.

\section{B. Topology Mismatching}

Studies in [25] have shown that only 2 to 5 percent of Gnutella connections link peers within a single autonomous system (AS). But more than 40 percent of all Gnutella peers are located within the top 10 ASes. This means that most Gnutella-generated traffic crosses AS borders so as to increase topology mismatching costs. The same message can traverse the same physical link multiple times, causing large amount of unnecessary traffic.

As we have discussed, the stochastic peer connection and peers randomly joining and leaving a P2P network can cause topology mismatching between the P2P logical overlay network and the physical underlying network. For example, Figure 3(a) is another subset of the P2P system of Figure 1. Figures 3(a) and 3(b) are two overlay topologies on top of the 
TABLE 1: TTL2-DETECTOR MESSAGE BODY

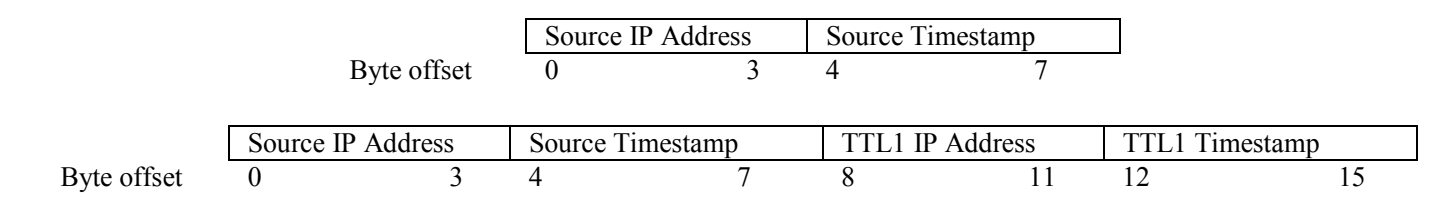

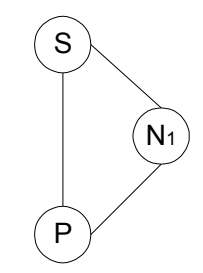

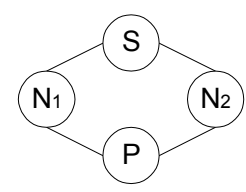

(b) (a)

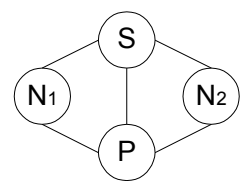

(c)
Figure 4. Peer $P$ receives $\mathrm{d}(i, S, v)$ multiple times

underlying physical topology shown in Figure 3(c). Suppose $\mathrm{C}_{1}$ and $\mathrm{C}_{3}$ are in the same autonomous system (AS), while $\mathrm{C}_{2}$ and $\mathrm{C}_{4}$ are in another AS. We assume that the physical link delay between $\mathrm{C}_{1}$ and $\mathrm{C}_{4}$ is much longer than all of the other links in Figure 3(c). Clearly, in the inefficient overlay of Figure 3(a), the query message from source $\mathrm{S}$ will traverse the longest link $\mathrm{C}_{1} \mathrm{C}_{4} 4$ times, which is a scenario of topology mismatching. If we can construct an efficient overlay shown in Figure 3(b), the message needs to traverse all the physical links in Figure 3(c) only once.

\section{Three Main Operations of LTM}

Optimizing inefficient overlay topologies can fundamentally improve P2P search efficiency. If the system can detect and disconnect the low productive logical connections such as $\mathrm{A}_{2} \mathrm{~A}_{3}$ and $\mathrm{B}_{1} \mathrm{~B}_{3}$ shown in Figure 2, and switch the connections of $\mathrm{C}_{1} \mathrm{C}_{2}, \mathrm{C}_{2} \mathrm{C}_{3}$ and $\mathrm{C}_{3} \mathrm{C}_{4}$ shown in Figure $3(\mathrm{a})$ to $\mathrm{C}_{3} \mathrm{C}_{1}$, $\mathrm{C}_{1} \mathrm{C}_{4}$ and $\mathrm{C}_{4} \mathrm{C}_{2}$ shown in Figure $3(\mathrm{~b})$, the total network traffic could be significantly reduced without shrinking the search scope of queries. This is the basic principle of our proposed location-aware topology matching technique. Three operations are defined in LTM: TTL2 detector flooding, low productive connection cutting, and source peer probing.

\section{1) TTL2-detector flooding}

Based on Gnutella 0.6 P2P protocol [4], we design a new message type called TTL2-detector. In addition to the Gnutella's unified 23-byte header for all message types, a TTL2-detector message has a message body in two formats as shown in Table 1. The short format is used in the source peer, which contains the source peer's IP address and the timestamp to flood the detector. The long format is used in a one-hop peer that is a direct neighbor of the source peer, which includes four fields: Source IP Address, Source Time- stamp, TTL1 IP Address, TTL1 Timestamp. The first two fields contain the source IP address and the source timestamp obtained from the source peer. The last two fields are the IP address of the source peer's direct neighbor who forwards the detector, and the timestamp to forward it. In the message header, the initial TTL value is 2 . The payload type of the detector can be defined as $0 \times 82$.

Each peer floods a TTL2-detector periodically. We use $\mathrm{d}(i, S, v)$ to denote the TTL2-detector who has the message ID of $i$ with TTL value of $v$, and is initiated by $S$. We use $\mathrm{N}(S)$ to denote the set of direct logical neighbors of $S$, and use $\mathrm{N}^{2}(S)$ to denote the set of peers being two hops away from $\mathrm{S}$. A TTL2-detector can only reach peers in $\mathrm{N}(S)$ and $\mathrm{N}^{2}(S)$. We use network delay between two nodes as a metric for measuring the cost between nodes. The clocks in all peers can be synchronized by current techniques in an acceptable accuracy $^{1}$. By using the TTL2-detector message, a peer can compute the cost of the paths to a source peer. As an example in Figure 4(a), when peer $P$ receives a d $(i, S, l)$, it can calculate the cost of link SP from Source Timestamp and the time $P$ receives the d $(i, S, l)$ from $S$. When $P$ receives a d $(i, S, 0)$, it can calculate the cost of link $\mathrm{SN}_{1}$ from TTL1 Timestamp and Source Timestamp, and $\mathrm{N}_{1} \mathrm{P}$ from TTL1 Timestamp and the time $P$ receives the $\mathrm{d}(i, S, 0)$ from $N_{l}$. As we can see in an inefficient overlay topology, the peers in set $\mathrm{N}^{2}(S)$ may receive $\mathrm{d}(i, S, v)$ more than once, such as peer $P$ in Figure 4(a)(c). If a peer receives $\mathrm{d}(i, S, v)$ multiple times, it will conduct the operations in the second step of LTM, low productive connection cutting.

\section{2) Low productive connection cutting}

There are three cases for any peer $P$ who receives d $i, S$, v) multiple times.

Case 1: $P$ receives both $\mathrm{d}(i, S, l)$ and $\mathrm{d}(i, S, 0)$ as shown in Figure 4(a). In this case, $\mathrm{d}(i, S, 1)$ comes from path $\mathrm{SP}$, while $\mathrm{d}(i, S, 0)$ comes from $\mathrm{SN}_{1} \mathrm{P}$. The costs of $\mathrm{SP}, \mathrm{SN}_{1}$, and $\mathrm{N}_{1} \mathrm{P}$ can be calculated from the timestamps recorded in $\mathrm{d}(i, S$, $0)$ and $\mathrm{d}(i, S, l)$. If $\mathrm{SP}$ or $\mathrm{N}_{1} \mathrm{P}$ has the largest cost among the three connections, $P$ will put this connection into its will-cut list that is a list of connections to be cut later. If $\mathrm{SN}_{1}$ has the largest cost, $P$ will do nothing. Note that LTM is fully distributed and all peers do the same LTM operations. In the case of $\mathrm{SN}_{1}$ having the largest cost, $\mathrm{N}_{1}$ will put this connection into $\mathrm{N}_{1}$ 's will-cut list. A peer will not send or forward queries to connections in its will-cut list, but these connec-

\footnotetext{
${ }^{1}$ Current implementation of NTP version 4.1.1 in public domain can reach the synchronization accuracy down to 7.5 milliseconds [7]. Another approach is to use distance to measure the communication cost, such as the number of hops weighted by individual channel bandwidth.
} 
tions have not been cut in order for query responses to be delivered to the source peer along the inverse search path.

Case 2: $P$ receives multiple $\mathrm{d}(i, S, 0) \mathrm{s}$ from different paths as shown in Figure 4(b). In LTM, $P$ randomly takes two of the paths, such as $\mathrm{SN}_{1} \mathrm{P}$ and $\mathrm{SN}_{2} \mathrm{P}$ in Figure $4(\mathrm{~b})$, to process at each time. Other paths, if any, will be handled in the next round of optimization. Thus, one important factor to affect the performance of LTM is the frequency for each peer to issue TTL2-detector messages. Our simulation results in Section V will show that the optimal LTM frequency is determined by the average peer lifetime and query frequency. Peer $P$ can calculate the costs of $\mathrm{SN}_{1}, \mathrm{SN}_{2}, \mathrm{~N}_{1} \mathrm{P}$ and $\mathrm{N}_{2} \mathrm{P}$. If $\mathrm{N}_{1} \mathrm{P}$ or $\mathrm{N}_{2} \mathrm{P}$ has the largest cost, $P$ will put it into its will-cut list. If $\mathrm{SN}_{1}$ or $\mathrm{SN}_{2}$ has the largest cost, $P$ will do nothing. As we have discussed above, $\mathrm{SN}_{1}$ or $\mathrm{SN}_{2}$ having the largest cost will be cut by one of the other three nodes.

Case 3: $P$ receives one $\mathrm{d}(i, S, 1)$ and multiple $\mathrm{d}(i, S, 0) \mathrm{s}$ as shown in Figure $4(\mathrm{c})$. In this case, $P$ will process the path receiving $\mathrm{d}(i, S, 1)$ and one path randomly selected from the multiple paths of $\mathrm{d}(i, S, 0) \mathrm{s}$ forming a scenario of Case 1 .

A connection in a will-cut list will be disconnected when it has been in the list for a certain time period. Our simulation results in Section $\mathrm{V}$ will show that a period of 50 seconds is optimal in a system with the average number of neighbors of 6 , peer average lifetime of 10 minutes, and query frequency of 0.3 queries per peer per minute. If a connection is cut by $P$, the IP address of the other node in this connection and the cost of the connection will be recorded in P's cut list. The cut list in a peer records the information of the connections disconnected by this peer and is designed to be used when the peer attempts to make a new connection so that the connections in the cut list will not be established again.

\section{3) Source peer probing}

For a peer $P$ who receives only one $\mathrm{d}(i, S, 0)$ during a certain time period (e.g., 10 seconds), and $P \in\left(N^{2}(S)-N(S)\right)$, it will try to obtain the cost of PS by checking its cut list first. If $S$ is not in the list, $P$ will probe the distance to $S$ (see Figure 5). After obtain the cost of PS, $P$ will compare this cost with the costs of $\mathrm{SN}_{1}$ and $\mathrm{N}_{1} \mathrm{P}$. If PS has the largest cost, $P$ will not keep this connection. Otherwise, this connection will be created. In the Internet, the cost of SP and the cost of PS may not be the same. We use the cost of PS to estimate the cost of SP.

Now let's look back the inefficient overlay topology shown in Figure 3(a). Figures 6(a)-(f) illustrate the process that LTM optimizes the overlay by using three operations discussed above.

\section{Traffic Overhead of LTM}

The simplicity of blind flooding makes it very popular in practice. This mechanism relays a query message to all its logical neighbors, except the incoming peer. For each query, each peer records the neighbors that relay the query to it. Therefore, in the worst case, the same query message can be sent on each logical link at most twice as illustrated in Figure

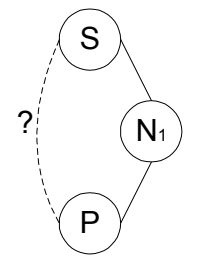

Figure 5. Source peer probing (a)

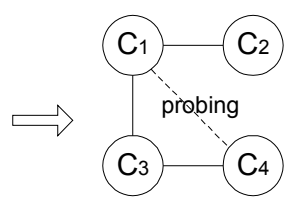

(c)

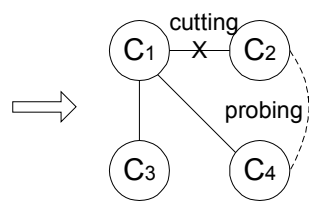

(e)

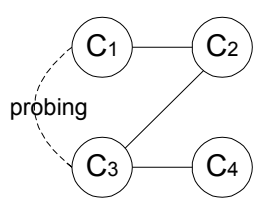

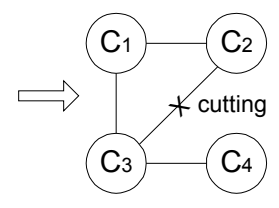

(b)

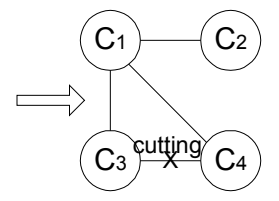

(d)

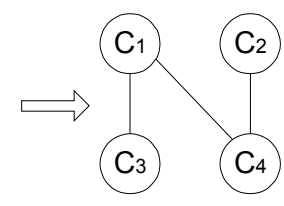

(f)
Figure 6. An example of LTM

1. For an overlay network with $n$ peers, we use $c_{n}$ to denote the average number of neighbors, and use $c_{e}$ to denote the average cost of the logical links. The total traffic caused by a query is less than or equal to $n c_{n} c_{e}$. In a typical P2P system, the value of $n$ (more than millions) is much greater than $c_{n}$ (less than tens) [31], so we can view both $c_{n}$ and $c_{e}$ as constant numbers. Thus, in the flooding-based search, the traffic incurred by one query from an arbitrary peer in a P2P network is $O(n)$. As observed in [32], each peer issues 0.3 queries per minute in average. Thus, the per minute traffic incurred by a P2P network with $n$ peers is $O\left(n^{2}\right)$.

Recall that each $\mathrm{d}(i, S, v)$ has a TTL value of 2 in a source peer. So the traffic for one time LTM optimization in all peers is at most $2 n c_{\mathrm{n}}{ }^{2} c_{\mathrm{e}}$. If each peer conducts LTM $k$ times per minute, the total traffic is $2 k n c_{n}{ }^{2} c_{e}$. Our simulation results will show that the best value for $k$ is 2 or 3 . Thus, the per minute traffic overhead incurred by LTM to the P2P network is $O(n)$.

Compared with the query traffic savings, the traffic overhead from LTM is trivial, which will be quantitatively shown in Section V.B. 
One question is why we don't use TTLj-detector with a TTL of $j>2$ in a source peer so that cycles with more than 4 links can be detected and broken. There are two reasons for not doing so. First, if $j>2$, the traffic caused by detector flooding will be increased significantly. Second, if the most expensive connection in a cycle is cut and its cost is not substantially larger than the costs of other links in the cycle, a query initiated from any of the two end peers in the broken cycle will need to traverse a path much more expensive than the cost on the cut connection to reach another end peer.

\section{Simulation Methodology}

We describe the three performance metrics we use in our simulations, our simulation setup, and parameter settings.

\section{A. Performance Metrics}

A well-designed search mechanism should seek to optimize both efficiency and Quality of Service (QoS). Efficiency focuses on better utilizing resources, such as bandwidth and processing power, while QoS focuses on userperceived qualities, such as number of returned results and response time. In unstructured $\mathrm{P} 2 \mathrm{P}$ systems, the QoS of a search mechanism generally depends on the number of peers being explored (queried), response time, and traffic overhead. If more peers can be queried by a certain query, it is more likely that the requested object can be found. So we use three performance metrics: average traffic cost versus search scope, average neighbor distance, and query response time.

Traffic cost is one of the parameters seriously concerned by network administrators. Heavy network traffic limits the scalability of P2P networks [26] and is also a reason why a network administrator may prohibit $\mathrm{P} 2 \mathrm{P}$ applications. We define the traffic cost as network resource used in an information search process of P2P systems, which is a function of consumed network bandwidth and other related expenses.

Search scope is defined as the number of peers that queries have reached in an information search process. Thus, with the same traffic cost, we aim to maximize the search scope; while with the same search scope, we aim to minimize the traffic cost.

Average neighbor distance $(D)$ is used to evaluate the optimization results of a logical topology. Let $D_{i}$ be the average delay between the source peer $i$ and all its logical neighbors. The value $D$ is defined as the average of all $D_{i}$ 's (i.e., all peers in the $\mathrm{P} 2 \mathrm{P}$ network). Minimizing average neighbor distance implies a better matching with the underlying physical network.

Response time of a query is one of the parameters concerned by P2P users. We define response time of a query as the time period from when the query is issued until when the source peer received a response result from the first responder.

\section{B. Simulation Setup}

To evaluate effectiveness of LTM, we first generate network topologies. Based on generated networks, we simulate P2P flooding search, host joining/leaving behavior, LTM, and index caching.

\section{1) Topology generation}

Two types of topologies, physical topology and logical topology, are generated in our simulation. The physical topology should represent the real topology with Internet characteristics. The logical topology represents the overlay P2P topology built on top of the physical topology. All P2P nodes are in a subset of nodes in the physical topology. The communication cost between two logical neighbors is calculated based on the physical shortest path between this pair of nodes. To simulate the performance of different search mechanisms in a more realistic environment, the two topologies must accurately reflect the topological properties of real networks in each layer.

Previous studies have shown that both large scale Internet physical topologies [34] and P2P overlay topologies [28] follow the small world and power law properties. Power law describes the node degree while the small world describes characteristics of path length and clustering coefficient [11]. The study in [28] found that the topologies generated using the AS Model have the properties of the small world and power law. BRITE [1] is a topology generation tool that provides the option to generate topologies based on the AS Model. Using BRITE, we generate 5 physical topologies each with 22,000 nodes. The logical topologies are generated with the number of peers (nodes) ranging from 2,000 to 8,000 . The average number of neighbors of each node is ranging from 4 to 10 .

We simulate LTM for all the generated logical topologies on top of each of the 5 generated physical topologies. We also simulate this approach in a real-world P2P topology (based on DSS Clip2 trace). We obtained consistent results on the real-world topology and the generated topologies. In order to show a thorough performance discussion, we only present our performance on various generated topologies.

\section{2) Flooding search simulation}

Our simulation is based on observed distributions. Content popularity of a publisher follows Zipf-like distribution (aka Power Law) $[8,10]$, where the relative probability of a request for the ith most popular page is proportional to $1 / i^{\alpha}$, with $\alpha$ typically taking on some value less than unity. The observed value of the exponent varies from trace to trace. The request distribution does not follow the strict Zipf's law (for which $\alpha=1$ ), but instead does follow a more general Zipflike distribution. Query word frequency does not follow a Zipf distribution $[16,35]$. User's query lexicon size does not follow a Zipf distribution [35] but with a heavy tail. Both the overall traffic and the traffic from $10 \%$ popular nodes are heavy-tailed in terms of the host connectivity, traffic volume, and average bandwidth of the hosts [31]. Studies in [30] have suggested a log-quadratic distribution $\left(10^{-\alpha^{2}}\right)$ for stored file locality and transfer file locality. The time length that nodes 
remain available follows a log-quadratic curve [30], which could be approximated by two Zipf distributions.

In our simulation, we simulate flooding search used in Gnutella network by conducting the Breath First Search algorithm from a specific node. A search operation is simulated by randomly choosing a peer as the sender, and a keyword according to Zipf distribution. In our first simulation, 100,000 search operations are simulated.

\section{3) A dynamic P2P environment}

P2P networks are highly dynamic with peers joining and leaving frequently. The observations in [31] have shown that over $20 \%$ of the logical connections in a P2P last 1 minute or less, and around $60 \%$ of the IP addresses keep active in FastTrack for no more than 10 minutes each time after they join the system. The measurement reported in [28] indicated that the median up-time for a node in Gnutella and Napster is 60 minutes. Studies in [9] have argued that measurement according to host IP addresses underestimates peer-to-peer host availability and have shown that each host joins and leaves a P2P system 6.4 times a day on average, and over $20 \%$ of the hosts arrive and depart every day. Although the numbers they provided are different to some extent, they share the same point that the peer population is quite transient. We simulate the joining and leaving behavior of peers via turning on/off logical peers. In our simulation, every node issues 0.3 queries per minute, which is calculated from the observation data shown in [32], i.e., 12,805 unique IP addresses issued $1,146,782$ queries in 5 hours. When a peer joins, a lifetime in seconds will be assigned to the peer. The lifetime of a peer is defined as the time period the peer will stay in the system. The lifetime is generated according to the distribution observed in [28]. The mean of the distribution is chosen to be 10 minutes [31]. The value of the variance is chosen to be half of the value of the mean. The lifetime will be decreased by one after passing each second. A peer will leave in next second when its lifetime reaches zero. During each second, there are a number of peers leaving the system. We then randomly pick up (turn on) the same number of peers from the physical network to join the overlay.

\section{4) Combining LTM with index cache}

To investigate whether LTM could be employed together with other approaches, we also simulate a strategy of combining LTM with a response index caching scheme, in which query responses are cached in passing peers along the returning path. In our simulation, each peer keeps a local cache and a response index cache. The size of a response index cache is bounded by 200 items. The average number of neighbors is 6 .

\section{PERformance EVAluation}

We present our simulation results in this section. Our simulation results on overlay networks of 2,000 nodes, 3,000 nodes, 5,000 nodes, and 8,000 nodes on top of 22,000-nodes
Internet-like physical networks are consistent. We only present the results based on the overlay network with 8,000 nodes.

\section{A. Effectiveness of LTM in Static Environment}

In our first simulation, we study the effectiveness of LTM in a static $\mathrm{P} 2 \mathrm{P}$ environment where the peers do not join and leave frequently. This will show that without changing the overlay topology, how many LTM optimization steps are required to reach a better topology matching.

1) Traffic cost vs. search scope

The goal of LTM scheme is to reduce traffic cost as much as possible while retaining the same search scope. Figure 7 compares the traffic cost incurred by the original Gnutellalike system and by the system after one-step LTM optimization. One-step means every peer makes LTM optimization only once.

In Figure 7 , the curve of ' $c_{n}$-neigh' shows the average traffic cost caused by a query to cover the search scope in $\mathrm{X}$ axis, where in the system the average number of logical neighbors is $c_{n}$. The dashed curves represent performance results without using LTM, while solid curves represent the results with LTM optimizations. Figure 7 shows that to cover the same search scope, one-step LTM reduces the traffic cost significantly, and the reduction rate increases as the search scope increases. In other words, with a given traffic cost, LTM will increase its search scope. Figure 8 shows that the traffic cost decreases when LTM is conducted multiple times, where the search scope is all 8000 peers. We can see that the traffic cost reduction reaches to a threshold after the second or third step LTM optimization. LTM can be convergent as fast as in 2-3 steps.

\section{2) Average neighbor distance and response time}

Average neighbor distance reflects effectiveness of LTM on topology matching. Figure 9 shows the average neighbor distance versus LTM optimization steps. Compared with the original Gnutella-like network without LTM scheme (0 optimization steps), one-step LTM optimization reduces average neighbor distance by about 55\%, and more steps of LTM may cut average neighbor distance to around $65 \%$.

Short query response time is always desirable in P2P systems. The simulation results in Figure 10 show that LTM can effectively shorten the query response time by about $62 \%$. The tradeoff between query traffic cost and response time has been discussed in [38]. P2P systems with a large number of average connections offer a faster search speed while increasing traffic. One of the strengths of LTM scheme is that it reduces both query traffic cost and response time without decreasing the query success rate. 


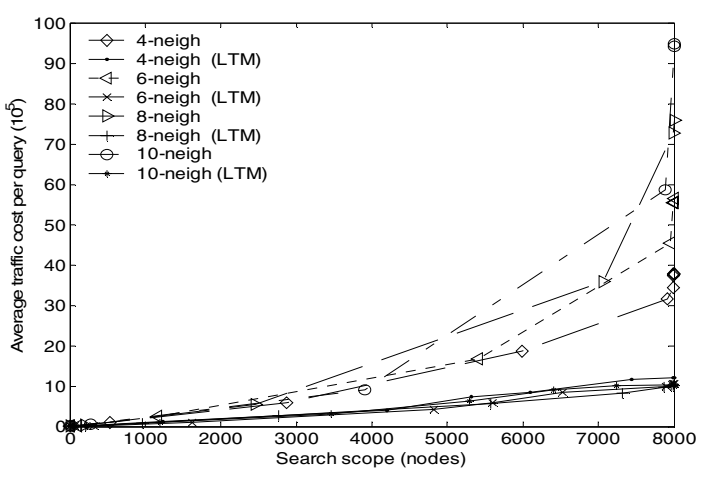

Figure 7. Traffic cost vs. search scope

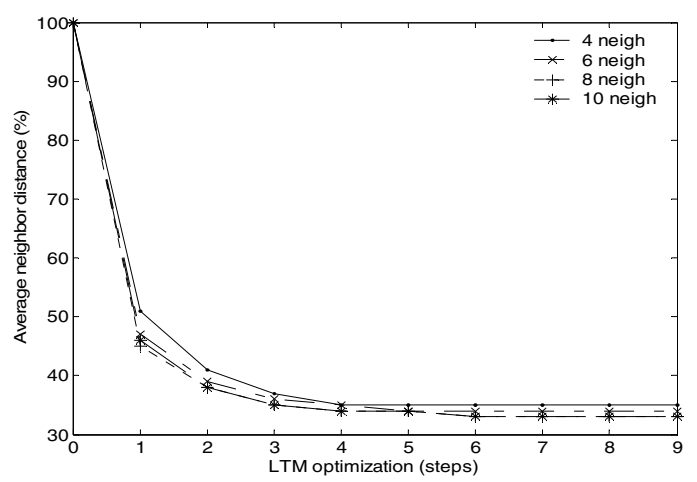

Figure 9. Average neighbor distance vs. optimization step

\section{B. LTM in Dynamic Environment}

We further evaluate the effectiveness of LTM in dynamic P2P systems and explore the best frequency for each peer to conduct LTM. We first discuss the performance impact of the will-cut list and the cut list. The average number of logical neighbors we use is 6 .

\section{1) Effectiveness of will-cut list ( $W$-C)}

From our simulation results in dynamic environments, we found that with the same search scope the query success rate in dynamic environments is decreased by about $5 \%$ compared with the static environment, as shown in Figure 11 (compare curves of static Gnutella-like and dynamic Gnutella-like). One extreme case is when the search scope is $100 \%$, which means that each query can reach all peers and we guarantee the query result is available in at least one of the peers. The search success rate is expected to be $100 \%$ in this case, but it is only $95 \%$. The reason of the $5 \%$ loss in query success rate is that the query responses cannot be returned due to peers' dynamic leaving behavior. We call this phenomena response loss problem.

If we don't use the will-cut list in LTM, a connection will be cut immediately when it is found to be low productive, which will cause a very serious response loss problem because many responses may not be returned due to the cut connections. The curve of LTM without $W-C$ in Figure 11 shows that the query success rate is significantly decreased by $30-40 \%$ without using the will-cut list. The LTM is conducted once every minute in this simulation. Retaining query success rate is

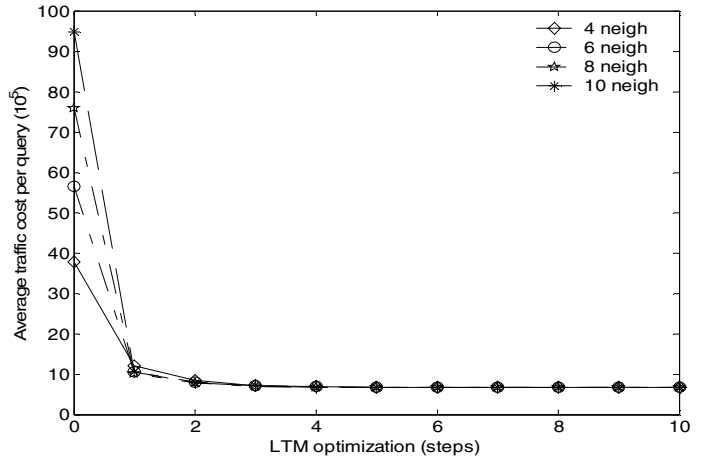

Figure 8. Traffic reduction vs. optimization step

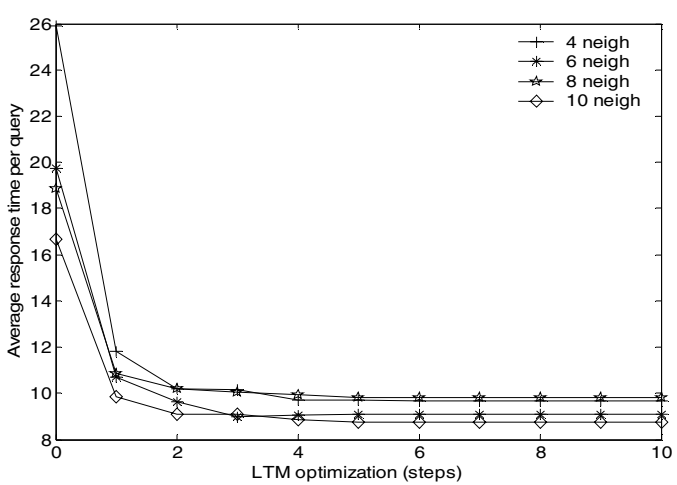

Figure 10. Average response time vs. optimization step

the reason we design the will-cut list, each of which can hold 20 connections in our simulation. The up to 20 low productive connections will not be used to forward queries, but only used to return query results. The lifetime of the connections in a will-cut list determines the query success rate. In Figure 11, a curve of $L T M$ with $W-C-n$ means the lifetime of a will-cut connection is $n$ seconds. We can see that the query success rate can be retained if the connections can be kept in the will-cut list for 50 seconds.

\section{2) Effectiveness of cut list}

If we don't use the cut list, a connection that has just been cut may be established again. Thus the LTM optimization rate will be limited. Figure 12 compares the overhead incurred by LTM with and without the use of the cut list. The fluctuations of the curves represent the dynamic nature of the network as time goes. The curve of $L T M-k$ means each peer conducts LTM for $k$ times per minute. We can see that the use of the cut list reduces traffic overhead by about 50\% compared with the case without using the cut list.

\section{3) Effectiveness and frequency of LTM}

We use the will-cut list and the cut list in this part of simulation. Compared with a Gnutella-like system, Figures 13 and 14 show the effectiveness of LTM on reducing average traffic cost and query response time. Since LTM adds some traffic overhead due to the TTL2 detector flooding, there exists an optimal frequency for each peer to conduct LTM independently. We simulate LTM in different frequencies ranging from $1 / 4$ to 4 times every minute. We consider a frequency to be 


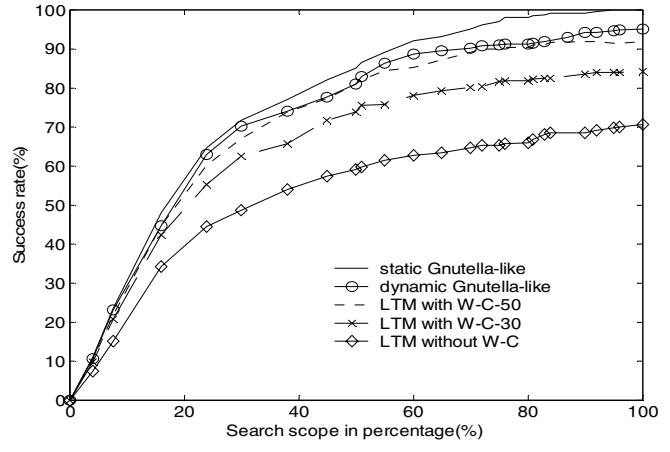

Figure 11. Effectiveness of will-cut list

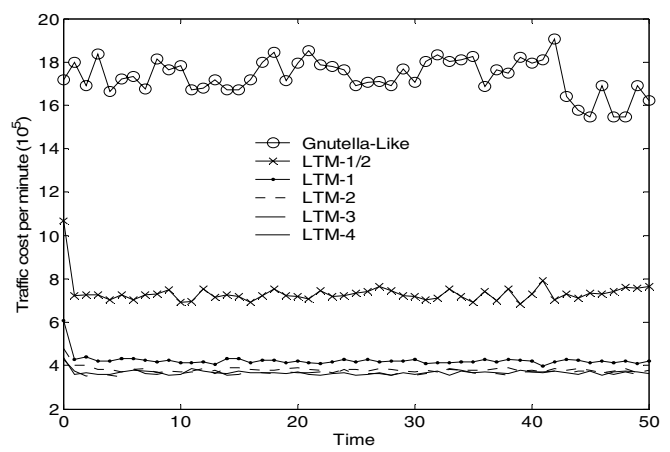

Figure 13. Total traffic vs. LTM frequeny

optimal if the next higher frequency does not increase the optimization by more than $3 \%$ compared with the current frequency. Results in Figures 13 and 14 show that under the assumption that peer average lifetime in a $\mathrm{P} 2 \mathrm{P}$ system is $10 \mathrm{~min}$ utes, and 0.3 queries are issued by each peer per minute, the optimal frequency for every peer to conduct LTM is twice per minute. With this frequency, about $75 \%$ reduction on traffic cost and $65 \%$ reduction on response time can be achieved. (average lifetime and query frequency) in our simulation. Figure 15 shows that LTM can be conducted less frequently if peer average lifetime is longer. Figure 16 shows that LTM should be conducted more frequently if more queries are issued. Both figures show that a larger average number of neighbors requires a higher LTM frequency.

\section{Combining LTM and Query Index Caching}

In this part, we evaluate a strategy of combining LTM with response index caching scheme. We compare the traffic cost and response time in a Gnutella-like system without any optimization, with query index caching only, with one-step LTM optimization only, and with one-step LTM optimization plus query index caching. Results in Figures 17 and 18 show that by combining LTM and query index caching the traffic cost is reduced by about 10 times without shrinking the search scope, and the average query response time is reduced by about 7 times.

\section{CONCLUSION AND FUTURE WORK}

We have evaluated our proposed location-aware topology matching, LTM, in both static and dynamic environments.

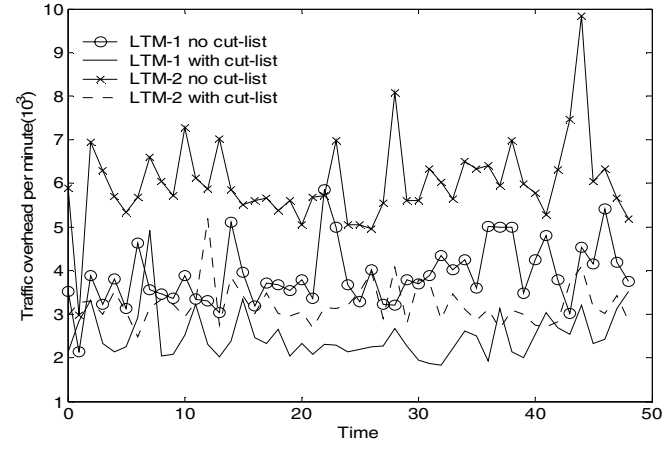

Figure 12. Effectiveness of cut list

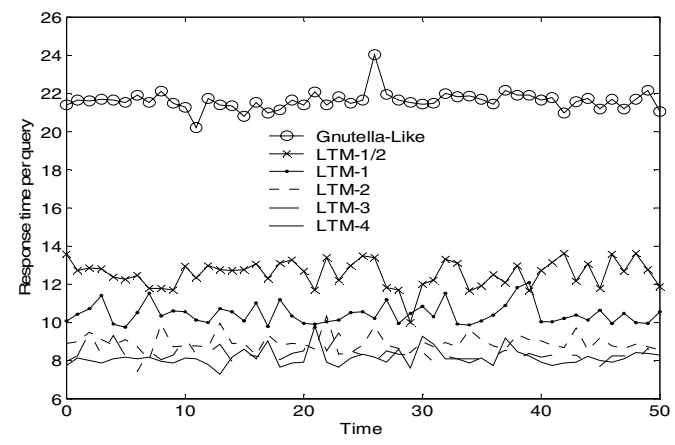

Figure 14. Response time vs. LTM frequency

Simulations in static P2P environments show that the significant performance benefit of LTM is consistent with various network sizes and average numbers of neighbors. In simulation studies of dynamic environments, we have investigated the optimal LTM frequency in a more realistic P2P environment. The results show that LTM achieves about $75 \%$ reduction on traffic cost and about $65 \%$ reduction on query response time. The impacts of peer average lifetime and query frequency on optimal LTM frequency have also been studied. We also show that our design of the will-cut list and the cut list can improve the performance of LTM. The ability that LTM can complement other advanced search approaches has been showed by a combination strategy of LTM with query index caching.

In LTM, each peer is aware of the location of other peers within a distance of two hops. The major advantage of LTM is that it is not only able to timely match the logical topology with the physical topology to significantly improve the search efficiency; it also guarantees to retain the search scope. This contribution makes LTM truly unique and highly effective. In addition, LTM is completely decentralized and scalable.

Future work on LTM will lead in two directions. One is to investigate the possibility of integrating LTM with other existing advanced search approaches to further improve search performance. The other one is to deploy and test an LTM prototype based on current version of Gnutella open source code in PlanetLab [23], an open, shared testbed for developing wide area network services. Note that the public domain Network Time Protocol (NTP) [7] can be embedded in our LTM to obtain an accurate timestamp measurement. 


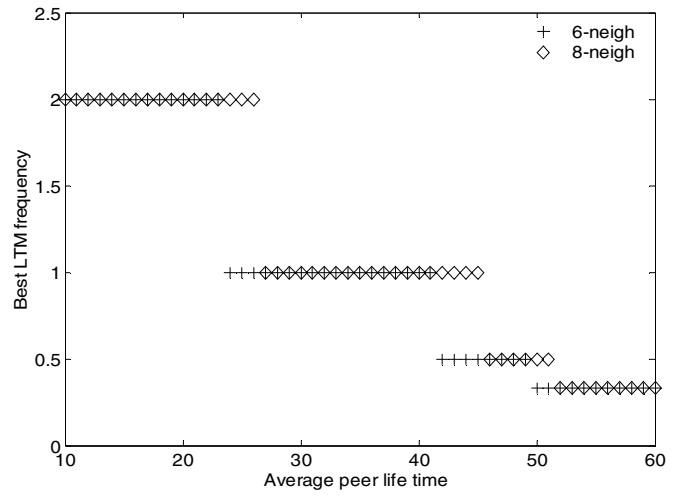

Figure 15. Optimal LTM frequency vs. average peer lifetime

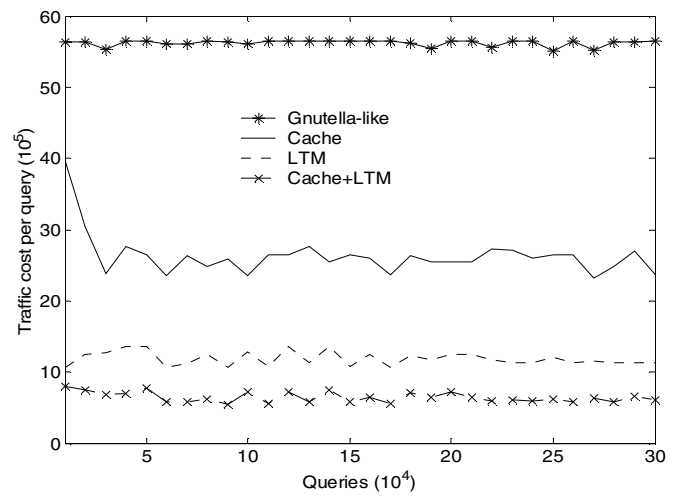

Figure 17. Traffic cost of four schemes

\section{REFERENCES}

[1] BRITE", http://www.cs.bu.edu/brite

[2] Fasttrack", http://www.fasttrack.nu

[3] Gnutella", http://gnutella.wego.com/

[4] The Gnutella protocol specification $0.6 "$, http://rfcgnutella.sourceforge.net

[5] KaZaA", http://www.kazaa.com

[6] Napster", http://www.napster.com

[7] NTP: The Network Time Protocol", http://www.ntp.org/

[8] V. Almeida, A. Bestavros, M. Crovella, and A. d. Olivera, "Characterizing Reference Locality in the WWW," in Proceedings of the IEEE Conference on Parallel and Distributed Information Systems (PDIS), 1996.

[9] R. Bhagwan, S. Savage, and G. M. Voelker, "Understanding Availability," in Proceedings of the 2nd International Workshop on Peer-to-Peer Systems(IPTPS'03), 2003.

[10] L. Breslau, P. Cao, L. Fan, G. Phillips, and S. Shenker, "Web Caching and Zipf-like Distributions: Evidence and Implications," in Proceedings of IEEE INFOCOM'99, 1999.

[11] T. Bu and D. Towsley, "On Distinguishing between Internet power law topology generators," in Proceedings of IEEE INFOCOM'02, 2002.

[12] Y. Chawathe, S. Ratnasamy, L. Breslau, N. Lanham, and S. Shenker, "Making Gnutella-like P2P Systems Scalable," in Proceedings of ACM SIGCOMM, 2003.

[13] Y. Chu, S. G. Rao, and H. Zhang, "A case for end system multicast," in Proceedings of ACM SIGMETRICS, 2000

[14] E. Cohen and S. Shenker, "Replication strategies in unstructured peerto-peer networks," in Proceedings of ACM SIGCOMM'02, 2002.

[15] O. D. Gnawali, "A Keyword-Set search system for peer-to-peer networks," Master's thesis, Massachusetts Institute of Technology, June 2002 .

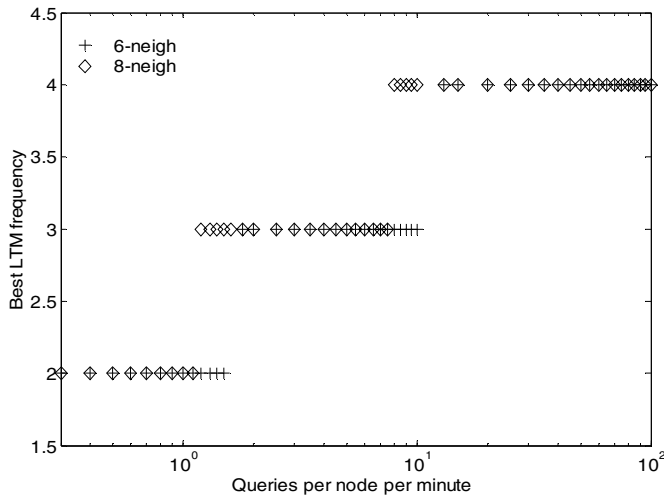

Figure 16. Optimal LTM frequency vs. average query frequency

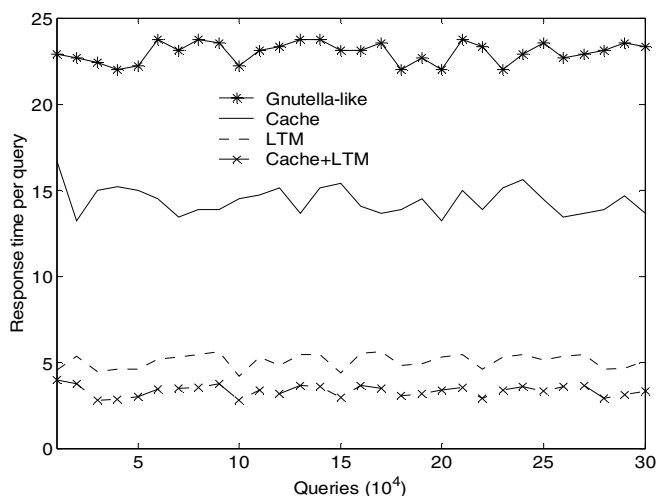

Figure 18. Average response time of four schemes

[16] B. J. Jansen, A. Spink, J. Bateman, and T. Saracevic, "Real Life Information Retrieval: a study of User Queries on the Web," SIGIR Forum, vol. 32, pp. 5-17, 1998

[17] B. Krishnamurthy and J. Wang, "Topology modeling via cluster graphs," in Proceedings of SIGCOMM Internet Measurement Workshop, 2001.

[18] Q. Lv, P. Cao, E. Cohen, K. Li, and S. Shenker, "Search and replication in unstructured peer-to-peer networks," in Proceedings of the 16th ACM International Conference on Supercomputing, 2002.

[19] E. P. Markatos, "Tracing a large-scale peer to peer system: an hour in the life of gnutella," in Proceedings of the 2nd IEEE/ACM International Symp. on Cluster Computing and the Grid, 2002.

[20] D. A. Menasce and L. Kanchanapalli, "Probabilistic Scalable P2P Resource Location Services," ACM SIGMETRICS Performance Evaluation Review, vol. 30, pp. 48-58, 2002.

[21] V. N. Padmanabhan and L. Subrananian, "An investigation of geographic mapping techniques for Internet hosts," in Proceedings of $A C M$ SIGCOMM'01, 2001

[22] S. Patro and Y. C. Hu, "Transparent Query Caching in Peer-to-Peer Overlay Networks," in Proceedings of the 17th International Parallel and Distributed Processing Symposium (IPDPS), 2003.

[23] L. Peterson, D. Culler, T. Anderson, and T. Roscoe, "A blueprint for introducing disruptive technology into the Internet," in Proceedings of HOTNETS, 2002.

[24] S. Ratnasamy, P. Francis, M. Handley, R. Karp, and S. Shenker, "A scalable content-addressable network," in Proceedings of $A C M$ SIGCOMM'01,2001.

[25] M. Ripeanu, A. Iamnitchi, and I. Foster, "Mapping the Gnutella Network," IEEE Internet Computing, 2002

[26] Ritter,"Why Gnutella can't scale. No, really", http://www.tch.org/gnutella.html

[27] A. Rowstron and P. Druschel, "Pastry: Scalable, distributed object location and routing for large-scale peer-to-peer systems," in Proceed- 
ings of International Conference on Distributed Systems Platforms, 2001.

[28] S. Saroiu, P. Gummadi, and S. Gribble, "A Measurement Study of Peer-to-Peer File Sharing Systems," in Proceedings of Multimedia Computing and Networking (MMCN), 2002.

[29] S. Saroiu, K. P.Gummadi, R. J. Dunn, S. D. Gribble, and H. M. Levy, "An Analysis of Internet Content Delivery Systems," in Proceedings of the 5th Symposium on Operating Systems Design and Implementation, 2002.

[30] M. T. Schlosser and S. D. Kamvar, "Availability and locality measurements of peer-to-peer file systems," in Proceedings of In proceedings of ITCom: Scalability and Traffic Control in IP Networks, 2002.

[31] S. Sen and J. Wang, "Analyzing peer-to-peer traffic across large networks," in Proceedings of ACM SIGCOMM Internet Measurement Workshop, 2002.

[32] K. Sripanidkulchai,"The popularity of Gnutella queries and its implications on scalability", 2.cs.cmu.edu/ kunwadee/research/p2p/gnutella.html

[33] R. M. Stoica, D. Karger, M. F. Kaashoek, and H. Balakrishnan, "Chord: A scalable peer-to-peer lookup service for Internet applications," in Proceedings of ACM SIGCOMM'01, 2001.

[34] H. Tangmunarunkit, R. Govindan, S. Jamin, S. Shenker, and W. Willinger, "Network Topology Generators: Degree-Based vs. Structural," in Proceedings of ACM SIGCOMM'02, 2002.

[35] Y. Xie and D. O'Hallaron, "Locality in Search Engine Queries and Its Implications for Caching," in Proceedings of IEEE INFOCOM'02, 2002.

[36] Z. Xu, C. Tang, and Z. Zhang, "Building topology-aware overlays using global soft-state," in Proceedings of International Conference on Distributed Computing Systems (ICDCS'03), 2003.

[37] B. Yang and H. Garcia-Molina, "Efficient search in peer-to-peer networks," in Proceedings of International Conference on Distributed Computing Systems (ICDCS'02), 2002.

[38] B. Y. Zhao, J. D. Kubiatowicz, and A. D. Joseph, "Tapestry: An infrastructure for fault-resilient wide-area location and routing," Technical Report UCB//CSD-01-1141, U.C.Berkeley 2001.

[39] Z. Zhuang, Y. Liu, L. Xiao, and L. M. Ni, "Hybrid Periodical Flooding in Unstructured Peer-to-Peer Networks," in Proceedings of International Conference on Parallel Processing(ICPP'03), 2003. 